\title{
The Pleiotropic Gene Theory of Senescence: Supportive Evidence from Human Genetic Disease
}

Roger L. Albin, M.D.

Department of Neurology, University of Michigan Medical Center

Senescence is a universal but poorly understood phenomenon among metazoans. One theoretically convincing but unproven evolutionary theory of senescence is the pleiotropic gene theory of Williams (1957). This paper develops the hypothesis that some human genetic diseases exemplify the type of phenotypic effects predicted by this theory. The evidence supporting this contention is reviewed and ways of testing this hypothesis are suggested. Other human genetic diseases could be examined in the same manner. Confirmation of this theory would have significant implications for the study of aging.

KEY WORDS: Senescence; Huntington's disease; Aging; Hemochromatosis; Peptic ulcer disease.

\section{INTRODUCTION}

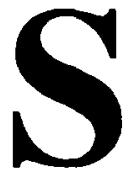

enescence may be defined as the postmaturity decline in survivorship and fccundity that accompanies advancing age (Rose et al. 1980). This phenomenon is an intrinsic property of metazoans and not the result of environmental hazard. Even in relatively optimal environments, all known animal species age and eventually die.

As pointed out by Williams (1957), senescence presents an apparent paradox. All adult metazoans are the result of a complex process of development and morphogenesis. It seems surprising that after attaining maturity, animals are unable to perform the apparently simpler task of maintaining somatic integrity. Natural selection would also seem to militate against senescence. The direct effect of natural selection would tend to favor individuals able to maintain their somatic vigor and reproductive potential the longest. Yet, most metazoans are relatively short-lived, and no potentially immortal animals have ever becn identified.

Several theories have been proposed to account for the existence of

Address reprint requests to: Roger L. Albin, M.D., Neuroscience Building, 1103 E. Huron St., Ann Arbor, MI 48104. 
senescence (for review, see Kirkwood 1985). Some theories sidestep this issue, presuppose that senescence is a fundamental feature of metazoans, and focus on defining variables that might account for the differences in life span among metazoans. Other theories appeal to the idea of group selection, and most focus on proximate mechanisms of senescence.

Probably the most rigorously developed theory of senescence is the pleiotropic gene theory. First proposed by Williams in 1957 (Williams 1957), it has been independently formulated by Wallace (1967) and extended by Hamilton (1966). While the theory theoretically satisfying, it has proved difficult to accumulate evidence for or against this theory. This paper develops the hypothesis that certain human genetic diseases exemplify the phenotypic effects predicted by the pleiotropic gene theory. Some aspects of this hypothesis have been independently put forward by Wallace (1971) and Wallace and Parker (1973), though they have not received much attention.

\section{THE PLEIOTROPIC GENE THEORY}

The pleiotropic gene theory rests on three assumptions. The first is that a given phenotypic effect of a gene may be restricted to a portion or portions of an organism's life history. The second assumption is that the impact on biologic fitness of any given gene is a function of the period(s) of the organism's life history during which the phenotypic effects are manifested. In general, the later in adult life a gene manifests itself, the less selective pressure it is exposed to. This occurs because the probability of individual survival falls with advancing age. This is true even in a population of potentially immortal individuals because environmental hazards will operate to remove individuals from the population. Consequently, a gene whose effects are felt relatively late in adult life is less likely to be exposed to selective pressures than one whose effects are felt at an earlier age simply because fewer individuals will survive to the age where the later onset gene has its effects. The third assumption is that a gene may be pleiotropic with respect to life history. That is, it may have one phenotypic effect during one portion of the life history and a different phenotypic during another portion of the life history.

Williams suggested that pleiotropic genes producing a deleterious effect at some age could be the beneficiary of favorable overall selection pressure if they also produced a different and positive effect on biologic fitness at an earlier age. The relative value of the beneficial effect will be amplified and the deleterious effect diminished by virtue of their relative positioning in the life history of the organism. When such a mutation occurs in a population of potentially immortal individuals, the pleiotropic allele will drive competing alleles to extinction. Once established, the pleiotropic senescence gene would reinforce the value of new mutations conferring relatively early benefit and thus make it likely that other senescence genes of this type would spread 
throughout the population. Hamilton (1966) has constructed models of this process and demonstrated that it could occur in primitive multicellular organisms. In the long history of life, such genes could have arisen on many occasions, and metazoans are likely to possess many loci producing deleterious late-life effects that originally conferred a selective advantage at earlier portions of the life span.

Unfortunately, obtaining direct confirmation of this theory has proven difficult. Senescence is rarely observed in wild animal populations, and obtaining the appropriate combination of demographic and genetic data in a wild population poses considerable difficulties. A recent study by Nesse (1988) of life table data for a wide variety of species showed that several species had a postmaturity acceleration of mortality rates, a finding predicted by the pleiotropic theory. Laboratory experiments using artificial selection in flour beetles (Sokal 1970; Mertz 1975) and drosophila (Rose and Charlesworth 1980; Luckinbill et al. 1984; Rose and Charlesworth 1981a,b) have also tended to support the theory but conflicting results exist (Lints 1985). These discrepancies are probably resolvable on the basis of differences in experimental technique, and the preponderence of the evidence favors the pleiotropic gene theory (Luckinbill and Clare 1985; Clare and Luckinbill 1985; Rose 1984). The supporting evidence consists of studies of genetic factors influencing longevity in selected strains of experimental animals. As yet, no one has isolated a specific allele or locus that satisfies the criteria of a pleiotropic senescence gene.

Humans would appear to be good subjects for the evaluation of the pleiotropic gene theory. Senescence is easily observable in humans, and a good deal is known about human genetics and demography. If the theory is correct, it should be possible to derive predictions from the theory that accord with some of the known features of human aging. Further, it may be possible to identify specific genes within human populations that cause morbidity and mortality while conferring a selective benefit at an earlier age.

\section{Pleiotropic Gene Theory Predictions, Human Aging, and Human Genetic Disease}

Several predictions follow from this theory that are relevant to human aging (Alexander 1982, 1987): (1) Maximum life span should remain limited despite optimization of the environment because life span will ultimately be limited by senescence genes. Despite the advent of modern medicine and public health measures, maximum human life span seems not to have increased over the period of time for which adequate documents exist (Fries 1980). (2) Among species, adult death rates from environmental hazard should be inversely correlated with maximum longevity because in species exposed to relatively hazardous environments, the selective pressures favoring genes with relatively early benefit will be marked. Humans, as compared with other species, face a relatively lower degree of environmental hazard and are 
among the most long lived of animals. (3) Where a sex difference exists, the sex that is exposed to a greater degree of environmental hazard should be more prone to senescence. In humans, adult males have a higher death rate from environmental hazard (especially intraspecies violence) and seem to be more prone to diseases of aging, especially atherosclerosis. (4) Senescence should result from the action of multiple genes and produce the dysfunction of multiple-cell types and organ systems because species are likely to accumulate multiple senescence genes over time. In the case of humans, this would correlate with the tendency of the aged to suffer from multiple chronic diseases and the gradual loss of functional reserve that occurs in all organ systems with human aging (Fries 1980).

Human genetic diseases provide the opportunity to search for specific allcles that might satisfy the definition of a pleiotropic senescence gene. Several human genetic diseases have their onset relatively late in adulthood and thus serve as experiments of nature with which to examine the pleiotropic gene theory. The identification of even one such allele as a pleiotropic senescence gene would provide strong supportive evidence for this theory of senescence. In addition, as the theory predicts that senescence genes are likely to be multiple and to affect multiple organ systems, we should be able to identify several different genetic diseases with different mechanisms of action that satisfy the definition of a pleiotropic senescence gene. A review of known adult onset human genetic diseases reveals three disorders that are good candidates for the role of pleiotropic senescence genes. As none of these disorders have been studied with the pleiotropic gene theory in mind, there is insufficient evidence to definitely identify any of them as pleiotropic senescence genes. Nonetheless, the evidence is suggestive and does identify these three diseases as promising candidates for evaluation of the pleiotropic gene theory.

\section{Huntington's Disease}

Huntington's disease (HD) is an autosomal dominant inherited neurodegenerative disorder characterized by progressive impairment of voluntary movement and increasing involuntary movements, cognitive decline, and personality disturbance (Martin et al. 1986). Prevalence in the United States is estimated at 30-45 per million (Conneally 1984). Age of onset is typically in the latter part of the third decade and early part of the fourth decade of life. Death usually follows 15-20 years after onset and is attendent upon the relentless course of the cognitive and motor impairments. Molecular genetic analysis of patients from widely dispersed pedigrees around the world has shown that this phenotype is invariably associated with a single gene located on chromosome number 4.

Virtually all clinicians who work with HD patients have been impressed hy the size of sibships in afflicted families. This has given rise to speculation that HD victims are more fecund and consequently possess greater biologic 
fitness than normals. Comparison of the fertility of HD victims with their unaffected siblings has revealed that in most surveyed areas HD victims produce more progeny than their unaffected sibs (Hayden 1981). The obvious objection is that this could result from diminished fertility of unaffected sibs rather than increased fertility of HD victims. Conflicting data exist on this point. Reed and Neel (1959) in their survey of HD in the state of Michigan (USA) and Shokeir (1975) in his survey of the provinces of Manitoba and Sasketchewan (Canada) have reported a depression of unaffected sib fertility as compared with the general population. On the other hand, Marx (1973), in her study of Minnesota (USA), and Stevens (1975) in the United Kingdom were unable to detect any differences between the fertility of unaffected sibs and the general population.

Five studies have attempted to compare the fertility of HD victims with that of the general population. One study revealed lower than average fertility (Recd et al. 1959), one showed essentially the same fertility as the general population (Wallace and Parker 1973), and three others (Marx 1973; Shokeir 1975; Stevens 1975) claimed increased fertility as compared with the general population. The mean of all studies shows a reproductive advantage of 1.09 over the general population. In the one study that revealed a diminished fertility relative to the general population, the authors concluded that diminished fertility was due in part to the premature termination of reproduction by institutionalization in mental hospitals. When the authors took this factor into account, relative fertility rose from 0.82 to 0.92 (the above calculation of mean reproductive advantage used the lower figure). This is of relevance because this study was conducted in the 1950s when institutionalization for psychiatric conditions was much more prevalent than it is today. Consequently, these fertility figures may be somewhat depressed because of a greater tendency to institutionalize patients with mild to moderate HD, a practice largely restricted to a few decades of modern history. In this study, case ascertainment was carried out to a large extent by review of the records of state-operated mental hospitals (Reed et al. 1958). At least one of the studies that found a reproductive advantage was based on a strenuous effort to identify all cases of HD within the study area, and it was not so dependent on the records of institutionalized patients (Shokeir 1975).

The preponderance of the evidence would seem to favor the notion that the HD gene increases the fertility of its carriers. One corollary of this conclusion is that the prevalence of $\mathrm{HD}$ might be increasing. This is difficult to ascertain because of a lack of suitable historic data. Hayden (1981) does remark that in comparing older with more recent epidemiologic studies, a rise in prevalence is noted, but this could easily be secondary to more complete ascertainment of cases in recent studies. However, in their survey of HD in south Wales (UK), Harper et al. (1979) were able to estimate that the birth rate of HD carriers has remained constant throughout the twentieth century. During the same time interval, the birth rate of the general popu- 
lation has dropped markedly, implying that the cohort-specific prevalence of HD may be increasing in their study population.

For unknown reasons, this apparent increase in the fertility of $\mathrm{HD}$ victims seems to be entirely due to heightened reproduction on the part of female HD victims. Men with HD seem to produce fewer offspring than normals and unaffected sibs. Female HD victims apparently also have a higher rate of bearing illegitimate children as compared with their unaffected sibs (Reed et al. 1958). Bird (1986) has speculated that the sex difference in fertility could be related to the excessive concentration of gonadotropin releasing hormone found in the hypothalami of female but not male HD victims.

It seems likely that the HD gene increases fertility while subsequently causing early mortality. As such, it would appear to be the type of pleiotropic gene envisioned by Williams. This must, however, remain a hypothesis for two reasons. First, while reproductive output is a valuable index of biologic fitness, a better measure would be the number of progeny who survive to the age of reproductive maturity. Family disruption is a common feature of HD families (Oliver 1970), and this could adversely affect biologic fitness. Reed and Neel (1959) concluded that the children of HD victims suffer from an increased infant mortality rate and that relative biologic fitness would be reduced by a factor of 0.015 . Second, while all studies comparing HD victim fertility with the general population have attempted to use age- and sexmatched controls, none has controlled for class or socioeconomic differences. HD victims tend to come from lower socioeconomic strata (Reed et al. 1958; Mattson 1974), and this could confound the interpretation of fertility data.

A study incorporating these demographic considerations should be possible. One excellent candidate for such a study would be the well studied Venezuela cohort (Young et al. 1986). This remarkably large (hundreds of individuals) and geographically stable pedigree has been instrumental in genetic and natural history studies of HD. Molecular genetic analysis of this pedigree had allowed the ascertainment of the genetic carrier status of all individuals in this pedigree. Histocompatibility typing has also been done, which would allow the determination of paternity. It should be possible to determine the fertility and progeny survival rates of gene and nongene carriers within this pedigree. Development of an appropriate demographic control population would allow determination of the biologic fitness of the HD gene.

\section{Idiopathic Hemochromatosis}

Idiopathic Hemochromatosis $(\mathrm{IH})$ is an autosomal recessive inherited disorder of iron metabolism (Bothwell et al. 1983). IH is one of the most common of human genetic diseases. Approximately $10 \%$ of the population of Europe and North America are heterozygous for the IH allele, and the prev- 
alence of homozygotes is estimated at $0.3 \%$ of the population. Penetrance, however, is usually incomplete. The mean age of onset of symptoms is approximately 50 with the vast majority of patients presenting between the ages of 40 and 60 (Finch and Finch 1955; Milder et al. 1980). IH is characterized by progressive deterioration in hepatic, endocrine pancreatic, cardiac, and pituitary function. Historically, untreated $\mathrm{IH}$ victims died within months after diagnosis. With the development of insulin therapy, the average period of survival was extended to four years, and the institution of therapeutic phlebotomy has extended the average survival period to 16 years (Milder et al. 1980). The molecular basis of the underlying defect is unknown, but IH is characterized by excessive absorption and abnormal distribution of iron. The capacity of humans to excrete iron is quite limited, and iron stores are largely regulated by control of intestinal iron absorption. IH victims have a dramatically heightened and unregulated capacity to absorb iron. They eventually saturate their normal iron storage capacity. Iron distribution then becomes abnormal, and progressive organ failure results. Even heterozygotes have a mildly increased capacity to absorb iron, although this does not result in pathologic sequealae (Cartwright et al. 1979).

The high prevalence of $\mathrm{IH}$ has lead to speculation that it may be the beneficiary of some positive selection pressure (Motulsky 1979; Rotter and Diamond 1987). The proposed advantage is that increased absorption of iron would help to combat the development of iron deficiency anemia. Such an advantage would be particularly marked for women during their reproductive years. Because of menstruation, pregnancy, and lactation, women tend to have smaller iron stores than men, and they are especially prone to develop iron deficiency anemia. An estimated $20 \%$ of women in the United States suffer from iron deficiency anemia, and this figure is much higher in developing countries where diets are poor in iron and hookworm infection is prevalent (Lee et al. 1980). The greater demands placed on iron stores in women probably explains why female homozygotes suffer serious organ impairment at a rate 5-10 times less than male homozygotes and that average onset of symptoms in women occurs a few years after average onset in men (Finch and Finch 1955). It would appear that the probable benefits of the IH allele accrue mainly to women and that the late life complications are reduced as well.

An alternative explanation for the high prevalence of the $\mathrm{IH}$ allele would be heterosis. Like sickle cell anemia, the bencfit to hetcrozygotes could outweigh the morbidity and mortality imposed upon homozygotes. As mentioned above, IH allele heterozygotes do have a mildly increased ability to absorb iron. This could provide them with a beneficial effect without incurring the penalty of developing clinical hemochromatosis. However, under conditions in which increased iron absorption is advantageous, the homozygotes would continue to have an advantage over both heterozygotes and normals. This would be especially true for women.

Evaluation of the biologic fitness of the $\mathrm{IH}$ allele could be carried out 
in areas where iron deficiency anemia is common. One could compare individuals, both heterozygotes and homozygotes for the $\mathrm{IH}$ allele, with matched controls and determine the prevalence of anemia within the two populations. One could also measure the reproductive success of IH allele hetero- and homozygotes to directly determine if this allele has a positive impact on biologic fitness. The IH gene is closely linked to the histocompatibility complex, and in caucasian populations the $\mathrm{IH}$ allele is closely associated with a particular HLA haplotype. IH allele carrier status could probably be assessed by iron studies or histocompatibility status or a combination of both.

\section{Pepsinogen 1 Related Peptic Ulcer Disease}

Peptic ulcer disease typically occurs in middle age. In one excellent population-based study, the median age of diagnosis was approximately 50, although the age specific incidence appears to rise linearly with age (Bonnevie 1975). The average age of onset may have been earlier some decades past (Eusterman and Balfour 1936). It is relatively common with a lifetime prevalence of at least 5\% in Europe (Grossman 1980), and in Denmark the incidence appears to be 1.4 new cases per 1,000 per year (Bonnevie 1975). In addition to causing considerable pain and disability, it is often life threatening because of massive gastrointestinal hemorrhage. Even with the excellent medical and surgical therapy presently available, victims of peptic ulcer disease have a small but significant decrease in life expectancy (Bonnevie 1978). This increased mortality seems to be especially pronounced among the elderly (Bonnevie 1978; Sonnenberg and Fritsch 1983). Several decades ago, the mortality rate was much higher with a nine-year cumulative mortality rate of $15 \%$ (Moutier 1932). Prior to the development of successful surgical techniques in the late nineteenth century, the mortality rate would have been much higher.

Several lines of evidence indicate that there is a substantial genetic component in susceptibility to peptic ulcer disease (Peterson and Rotter 1983). One well characterized genetic syndrome that predisposes to peptic ulcers is the overproduction of pepsinogen 1 (PG1) (Rotter et al. 1979a; Rotter et al. 1979b). This protein is a precursor to the proteolytic enzyme pepsin and is secreted into the stomach in the course of digestion. Excessive production of PG1 is inherited in an autosomal dominant manner. Hypersecretors of PG1 can also be identified by an elevated concentration of PG1 in serum. In some series, hypersecretion of PG1 is present in $50 \%$ of peptic ulcer disease patients (Samloff et al. 1975a; Rotter et al. 1979b), although not all individuals with elevated PG1 develop ulcers. The hypersecretion of PG1 is accompanied by excessive production of acid by the gastric mucosa (Samloff et al. 1975b; Rotter et al. 1979b).

Rotter and his colleagues (Rotter and Diamond 1987; Peterson and Rotter 1983) have speculated that gastric hyperacidity may confer some selective 
advantage because gastric acidity appears to form a nonspecific barrier to infection. This is especially important in the case of enteric infections and also may be a factor in resistance to tuberculosis. Decreased gastric acid secretion predisposes to cholera infection (Nalin et al. 1978). Individuals who have undergone partial gastric resection generally have reduced acid secretion and an increased susceptibility to tuberculosis (Balint 1958). Rotter et al. have pointed out that the putative benefits of gastric acid hypersecretion would have been especially marked in nineteenth-century Europe when tuberculosis was a leading cause of death and morbidity. These authors have further speculated that the selective pressure exerted by tuberculosis could have accounted for the marked rise in prevalence of peptic ulcer seen in the first half of the twentieth century (Ivey 1950).

This hypothesis could be tested in an area where tuberculosis and/or cholera are still prevalent. PG1 hypersecretors could be identified by measurement of serum PG1 levels, and the incidence rate of tuberculosis and/ or cholera in hypersecretors and normals could be compared.

\section{CONCLUSIONS}

This article has explored the possibility that study of human aging and human genetic diseases may allow verification of the pleiotropic gene theory of senescence. Predictions derived from the pleiotropic gene theory do accord with some of the known facts about human aging. In addition, certain human adult onset genetic diseases may exemplify the type of phenotypic effects predicted by the pleiotropic gene theory. While none of these genes can be definitively identified as a pleiotropic senescence gene, the available evidence is suggestive. All of these alleles may increase biologic fitness early in adult life and certainly cause increased mortality and morbidity later in life. Also in keeping with the pleiotropic gene theory is the fact that these candidate alleles occupy distinct loci and exert their deleterious effects on different organs and through different pathophysiologic mechanisms.

As discussed above, it should be possible to evaluate the biologic fitness of each of these alleles. Ultimately, one would wish to exclude the possibility of a transient polymorphism by following a study population for many generations to determine if there is an increasing frequency of the candidate allele within that population. However, in the absence of good historic information, it would be impossible to conduct such a longitudinal study in humans. This is especially true for industrialized countries because modern medicine has altered the natural history of diseases like IH and peptic ulcer disease. Consequently, the approach outlined above is probably the optimal one for the identification and evaluation of candidate human senescence genes. Several other adult onset human genetic diseases could also be evaluated as candidate pleiotropic senescence genes. This could include such common disorders as familial Alzheimer's disease, atherosclerotic heart dis- 
ease, and adult onset diabetes mellitus. Indeed, it has already been suggested that the alleles for adult onset diabetes mellitus have may conferred a selective advantage in preindustrial societies by optimizing carbohydrate metabolism under starvation conditions (Neel 1982).

The identification of even one gene or allele as a pleiotropic senescence gene would be strong evidence in favor of this theory of senescence. The consequences for gerontologic research and care of the elderly could be profound. The pleiotropic gene theory predicts that life span is genetically limited and that senescence is the probable product of multiple genes. Consequently, present efforts to define a single proximate mechanism of senescence and to manipulate that mechanism in the hope of extending life span would be doomed to failure and thus represent a waste of resources. Likewise, if aging and ultimately mortality are the result of a multiplicity of genetic effects, heroic efforts to prolong the lives of the elderly will be ultimately fruitless.

I would like to thank Nili Tannenbaum, Jang-Ho Cha, Jack Penney, Anne Young, and Richard Alexander for useful discussions on the topic of this paper. I am particularly grateful to Drs. Young and Alexander for critical readings of an earlier version of this manuscript. I also thank Suyin Liang for typing. This manuscript was completed while I was supported by NIH Training Grant 5-T32-NS07222-05.

\section{REFERENCES}

Alexander, R.D. Darwinism and Human Affairs, Seattle: University of Washington, 1982.

The Biology of Moral Systems, Hawthorne, New York: Aldine de Gruyter, 1987.

Balint, J.A. Pulmonary tuberculosis and partial gastrectomy. Gastroenterologia 90: 65-84, 1958. Bird, E.D. Huntington's chorea: Etiology and pathogenesis. In Handbook of Clinical Neurology: Extrapyramidal Disorders, Vol. 49, P.J. Vinken, G.W. Bruyn, H.L. Klawans (Eds.). New York: Elsevier, 1986, pp. 253-265.

Bonnevie, O. The incidence of duodenal ulcer in Copenhagen county. Scandinavian Journal of Gastroenterology 10: 385-393, 1975.

Survival in peptic ulcer. Gastroenterology 75: 1055-1060, 1978.

Bothwell, T.H., Charlton, R.W., Motulsky, A.G. Hemochromatosis. In The Metabolic Basis of Inherited Disease, J.B. Stanbury, J.B. Wyngaarden, D.S. Frederickson, J.L. Goldstein, M.S. Brown (Eds.). New York: McGraw-Hill, 1983, pp. 1269-1298.

Cartwright, G.E. Hereditary hemochromatosis: Phenotypic expression of the disease. New England Journal of Medicine 301: 175-179, 1979

Clare, M.J., Luckinbill, L.S. The effects of gene-environment interaction on the expression of longevity. Heredity 55: 19-29, 1985.

Conneally, P.M. Huntington's disease: Genetics and epidemiology. American Journal of Human Genetics 36: 506-526, 1984.

Eusterman, G.B., Balfour, D.C. The Stomach and Duodenum, Philadelphia: W.B. Saunders, 1936.

Finch, S.C., Finch, C.A. Idiopathic hemochromatosis, an iron storage disease. Medicine 34: $381-430,1955$

Fries, J.F. Aging, natural death and the compression of morbidity. New England Journal of Medicine 303: 130-135, 1980.

Grossman, M.I. Peptic ulcer: Definition and epidemiology. In Genetics and Heterogencity of 
Common Gastrointestinal Disorder, J.I. Rotter, I.M. Samloff, D.L. Rimoin (Eds.). New York: Academic Press, 1980, pp. 21-29.

Hamilton, W.D. The moulding of senescence by natural selection. Journal of Theoretical Bislogy 12: 12-45, 1966.

Harper, P.S., Walker, D.A., Tyler, A., Newcombe, R.G., Davies, K. Huntington's chorea: The basis for long-term prevention. Lancet II: 346-349, 1979.

Hayden, M.R. Huntington's Chorea, Berlin: Springer-Verlag, 1981.

Ivey, A.C. Peptic Ulcer Disease, Philadelphia: Blakiston, 1950.

Kirkwood, T.B.L. Comparative and evolutionary aspects of longevity. In Handbook of the Biology of Aging, New York: Van Nostrand Reinhold, 1985, pp. 27-44.

Lee, G.R., Wintrobe, M.M., Bunn, H.F. Iron deficiency anemia and the sideroblastic anemias. In Harrison's Principles of Internal Medicine, K.J. Isselbacher, R.D. Adams, E. Braunwald, R.G. Petersdorf, J.D. Wilson (Eds.). New York: McGraw-Hill, 1980, pp. 1514-1518.

Lints, F.A. Insects. In Handbook of the Biology of Aging, New York: Van Nostrand Reinhold, 1985, pp. 146-172.

Luckinbill, L.S., Clare, M.J. Selection for life span in Drosophila melanogaster. Heredity 55: 9-18, 1985.

Arking, R. Clare, M.J., Cirocco, W.C., Buck, S.A. Selection for delayed senescence in Drosophila melanogaster. Evolution 38: 996-1003, 1984.

Martin, J.B., Gusella, J.F. Huntington's disease: Pathogenesis and management. New England Journal of Medicine 315: 1267-1276, 1986.

Marx, R.N. Huntington's chorea in Minnesota. Advances in Neurology 1: 237-243, 1973.

Mattson, B. Huntington's chorea in Sweden. II. Social and clinical data. Acta Psychiatrica Scandinavica Supplementum 255: 221-235, 1974.

Mertz, D.B. Senescent decline in flour beetle strains selected for early adult fitness. Physiological Zoology 48: 1-23, 1975.

Milder, M.S., Cook, J.D., Stray, S., Finch, C.A. Idiopathic hemochromatosis, an interim report. Medicine 59: 34-49, 1980.

Motulsky, A.G. Letter: Genetics of hemochromatosis. New England Journal of Medicine 301: $1291,1979$.

Moutier, F., Quoted in Eusterman, G.B. and Balfour, D.C. The Stomach and Duodenum, Philadelphia: W.B. Saunders, 1936; p. 274.

Nalin, D.R., Levine, R.J., Levine, M.M., Hoover, D., Berquist, E., McLaughlin, J., Libonati, J., Alam, J., Hornick, R.B. Cholera, non-vibrio cholera, and stomach acid. Lancet II: 856-859, 1978.

Neel, J.V. The thrifty genotype revisited. In The Genetics of Diabetes Mellitus, J. Kobberling, R. Tattersall (Eds.). New York: Academic Press, 1982, pp. 283-293.

Neese, R.M. Life table tests of evolutionary theories of senencence. Experimental Gerontology, in press.

Oliver, J.E. Huntington's chorea in Northamptonshire. British Journal of Psychiatry 116: 241$253,1970$.

Petersen, G.M., Rotter, J.I. Genetic and evolutionary implications in peptic ulcer disease. American Journal of Physical Anthropology 62: 71-79, 1983.

Reed, T.E., Chandler, J.H., Hughes, E.M., Davidson, R.T. Huntington's chorea in Michigan. 1. Demography and genetics. Am. J. Hum. Genet. 10; 201-225, 1958.

- - Neel, J.V. Huntington's chorea in Michigan. 2. Selection and mutation. Am. J. Hum. Genet. 11: 107-136, 1959.

Rose, M.R., Charlesworth, B. A test of evolutionary theories of senescence. Nature 287: 141$143,1980$.

- Charlesworth, B. Genetics of life history in Drosophila melanogaster. I. Sib analysis of adult females. Genetics 97 : 173-186, $1981 \mathrm{a}$.

- Charlesworth, B. Genetics of life history in Drosophila melanogaster II. Exploratory selection experiments. Genetics 97: 187-196, 1981 b.

_- Laboratory evolution of postponed senescence in Drosophila melanogaster. Evolution 38: 1004-1010, 1984.

Rotter, J.I., Sones, J.Q., Samloff, I.M. Duodenal ulcer disease associated with elevated serum pepsinogen I, an inherited autosomal dominant disorder. New England Journal of Medicine 300: 63-66, 1979a. 
Peterson, G., Samloff, I.M., McDonnell, R.B., Ellis, A., Spence, M.A., Rimoin, D.L. Genetic heterogeneity of familial hyperpepsinogenemic I and normopepsinogenemic I duodenal ulcer disease. Annals of Internal Medicine 91: 372-377, 1979b.

- Diamond, J.M. News and Views: What maintains the frequencies of human genetic diseases? Nature 329: 289-290, 1987.

Samloff, I.M., Liebman, W.M., Panitch, N.M. Serum group I pepsinogen levels by radioimmunoassay in control subjects and patients with peptic ulcer. Gastroenterology 69: 83-90, 1975a.

--, Secrist, D.M., Passaro, E. A study of the relationship between serum group I pepsinogen levels and gastric acid secretion. Gastroenterology 69: 1196-1200, 1975 b.

Shokeir, M.H.K. Investigations on Huntington's disease in the Canadian prairies. II. Fecundity and fitness. Clin. Genet. 11: 349-353, 1975.

Sokal, R.R. Senescence and genetic load: Evidence from tribolium. Science 167: 1733-1734, 1970.

Sonnenberg, A., Fritsch, A. Changing mortality of peptic ulcer disease in Germany. Gastroenterology 84: 1553-1557, 1983.

Stevens, D.L.: Quoted in Hayden, M.R. Huntington's Chorea, Berlin: Springer-Verlag, 1981.

Wallace, D.C. The inevitability of growing old. Journal of Chronic Diseases 20: 475-486, 1967.

- , Huntington's chorea: A partial model of the aging process. Medical Journal of Australia 2: 1275-1276, 1971 .

- Parker, N. Huntington's chorea in Queensland: The most recent story. Advances in Neurology 1: 223-236.

Williams, G.C. Pleiotropy, natural selection, and the evolution of senescence. Evolution 11: 398-411, 1957.

Young, A.B., et al. Huntington's disease in Venezuela: Neurologic features and functional decline. Neurology 36: 244-249, 1986. 